\title{
Predicting Athlete Ground Reaction Forces and Moments from Spatio-temporal Driven CNN Models
}

\author{
William R. Johnson, Student Member, IEEE, Jacqueline Alderson, David Lloyd, and Ajmal Mian
}

\begin{abstract}
The accurate prediction of 3D ground reaction forces and moments (GRF/Ms) outside the laboratory setting would represent a watershed for on-field biomechanical analysis. To extricate the biomechanist's reliance on ground embedded force plates, this study sought to improve on an earlier Partial Least Squares (PLS) approach by using deep learning to predict 3D GRF/Ms from legacy marker-based motion capture sidestepping trials, ranking multivariate regression of GRF/Ms from five convolutional neural network (CNN) models. In a possible first for biomechanics, tactical feature engineering techniques were used to compress space-time and facilitate fine-tuning from three pre-trained CNNs, from which a model derivative of ImageNet called 'CaffeNet' achieved the strongest average correlation to ground truth GRF/Ms $r\left(F_{\text {mean }}\right) \mathbf{0 . 9 8 8 1}$ and $r\left(M_{\text {mean }}\right) \mathbf{0 . 9 7 1 5}$ ( $r R M S E 4.31$ and $7.04 \%$ ). These results demonstrate the power of CNN models to facilitate real-world multivariate regression with practical application for spatio-temporal sports analytics.
\end{abstract}

Index Terms-Biomechanics, Supervised learning, Image motion analysis, Pattern analysis.

\section{INTRODUCTION}

Conventional methods to generate GRF/Ms data required for the accurate estimation of joint forces andloads are confined to biomechanics laboratories far removed from the sporting field of play. This has been an ongoing frustration for sports biomechanists, who must forego the eco-logical validity of field-based data collections and manage the constraints of synthetic laboratory environments to accurately model musculoskeletal loading parameters (Figure 1) [1], [2]. In the laboratory, biomechanists commonly rely on gold standard marker-based passive retro-reflective systems which utilize high-speed modified video cameras (up to 2,000 Hz) that project strobes of infrared (IR) light onto small spherical retroreflective markers attached to a participant's body [1], [3]. The typical error of such systems for dynamic sporting movement is cited at $<2 \mathrm{~mm}$ [4]. Alongside motion data, it is common practise to capture synchronized GRF/Ms (shear

W. R. Johnson is with the School of Human Sciences (Exercise and Sport Science), The University of Western Australia, Perth Australia (e-mail: bill.johnson@uwa.edu.au).

J. Alderson is with the School of Human Sciences (Exercise and Sport Science), The University of Western Australia, Perth Australia, and the Sports Performance Research Institute New Zealand (SPRINZ), Auckland University of Technology, Auckland, New Zealand.

D. Lloyd is with the Menzies Health Institute Queensland, and the School of Allied Health Sciences, Griffith University, Gold Coast, Australia.

A. Mian is with the School of Computer Science and Software Engineering, The University of Western Australia, Perth, Australia.

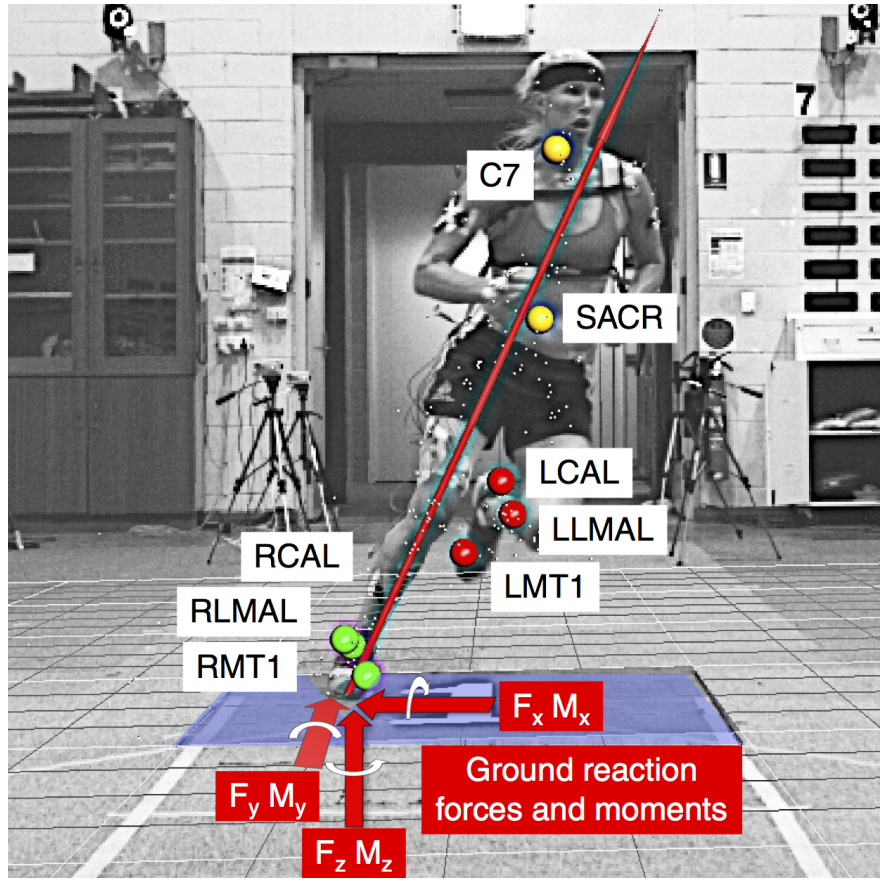

Fig. 1. Laboratory motion and force plate data capture overlay. The eight labeled markers used are shown artificially colored and enlarged, and visible through the body. The force plate is highlighted blue, and the ground reaction forces and moments depicted.

forces $F_{x}$, and $F_{y}$, vertical force $F_{z}$, and their corresponding rotation moments $M_{x}, M_{y}$ and $M_{z}$ ) as measured by transducers located in the four corners of the force platform (Advanced Mechanical Technology Inc., Watertown, MA, USA).

An increasingly popular approach to on-field data capture is to instrument the player with wearable sensors, with early examples of basic in-shoe pressure sensors and instrumented force shoes [5], [6], [7] evolving to current athletes wearing inertial sensors on multiple body segments [8], [9], [10]. However, the linear modeling applied to the sensor outputs from current wearable devices tends to overfit a particular movement (e.g. simple gait), or favor the vertical force $\left(F_{z}\right)$ component only [11].

From a sports injury perspective, rupture of the anterior cruciate ligament (ACL) can be one of the most serious for the community and professional athlete [12], with up to a reported $80 \%$ incidence rate being non-contact in nature, and 
$80 \%$ of these events occurring during a sidestep or singleleg landing maneuver [13], [14]. Studies cite elevated knee joint moments (KJMs) as a primary indicator of ACL injury risk [15], [16], and given that the most common approach to estimating KJMs is via inverse dynamics methods that require GRF/Ms input, data of these type are an obvious candidate for further investigation.

Previous computer vision and data science researchers have attempted to estimate GRF/Ms, however studies suffer from poor validation to ground truth data, are not sports related [17], [18], [19], require a full body modeling protocol with multiple inputs [20], or as before, predict only unidirectional GRF components (e.g. vertical $F_{z}$ ) [21]. Further, machine learning approaches in biomechanics appear to be limited to simple models and small sample sizes [22], [23], [24], [25].

Prior to this study, our team developed a prototype using a class of supervised multivariate regression, PLS [26], [27], chosen because of its characteristic to perform well with many predictor variables but limited samples. PLS works by projecting to a lower dimensional space where the covariance between predictor and response variables is maximized, and more recently, sparse PLS techniques have emerged which can better deal with multivariate responses when some of the predictor variables are noisy [28].

The success by Johnson et al. [27] of the initial PLS study encourages further investigation employing deep learning techniques, in particular CNN variants under frameworks such as Caffe (Convolutional Architecture for Fast Feature Embedding), TensorFlow and Torch [29], [30], [31]. The origins of many current deep learning approaches can be traced to recent ImageNet Large Scale Visual Recognition Challenges, and hence their strength in computer vision feature classification [32], [33]. Despite the fact motion capture is, by definition, spatio-temporal in nature, fine-tuning of existing image-based models such as AlexNet, CaffeNet, and GoogLeNet provides an opportunity to leverage training at scale, only needing to re-train selected higher-level components [34].

The contribution of this study is to investigate if pretrained CNN models can be transferred to improve 3D GRF/M predictions beyond what was achieved by PLS, using a subset of marker trajectories extracted from legacy motion capture sidestepping trials. The accuracy and validity of the approach was assessed by comparing mean alignment between GRF/Ms derived from ground truth force plate data against those predicted by a series of new models, ranked by CNN method. It was hypothesized that fine-tuning of a pre-trained CNN model would outperform previous PLS accuracy results, particularly in the case of GRMs which demonstrate greater noise and non-linearity.

\section{METHODS}

\section{A. Design and setup}

This study was made possible by access to the motion capture trial archive of The University of Western Australia (UWA), in which data capture sessions were carried out in multiple laboratories over a 17-year period from 2001-2017 (Figure 2). No new data capture was undertaken. Most research in the laboratory is sport-based, therefore all data included in this study was drawn from a young healthy athletic population aged between 16-35 years. Over this period, the number and type of infrared video cameras have changed (12-20 Vicon type MCam2, MX13, and T40S cameras; Oxford Metrics, Oxford, UK), as has the software to drive them (Workstation v4.6 to Nexus v2.5). However, selecting force platforms by specific types, as defined by the 'coordinate 3D' c3d file format (Motion Lab Systems, Baton Rouge, LA), meant the imported analog GRF/M data structures remained consistent. The force platforms in these laboratories are subject to annual calibration testing [35], therefore the source ground truth data was expected to contain a systematic error of the order GRF $1.4 \%$ and GRM $1.6 \%$. The customized full-body UWA marker set has also evolved during this time to range from 24-67 markers. Pilot research revealed that a subset of only eight of these passive retro-reflective markers (C7; sacrum SACR; plus hallux MT1, calcaneus CAL, and lateral ankle malleolus LMAL of each foot) were required to maximize trial inclusion, relevance to the sidestep movement input, and GRF/M data output (Figure 1] [36].

\section{B. Data preparation}

The total $\mathrm{c} 3 \mathrm{~d}$ file archive at the time of this study contained 433,186 trials. Under UWA ethics approval RA/4/1/8415, processing of this data was conducted using MATLAB R2017b (MathWorks, Natick, MA) in conjunction with the Biomechanical ToolKit 0.3 [37], Python 2.7 (Python Software Foundation, Beaverton, OR) and R 3.4.3 [38], running on Ubuntu v16.04 (Canonical, London, UK). Desktop PC hardware included a Core i7 4GHz CPU, 32GB RAM, and NVIDIA Titan X GPU (NVIDIA Corporation, Santa Clara, CA).

The data preparation phase was designed to avoid gaps or errors from the motion capture marker trajectories and force plate analog channels contaminating CNN model training [4], [35]. The eight nominated marker trajectories were required to be contiguous and labeled (Figures 3 \& fig_tbme_supp), and force plate channel data fully present, both for one complete stance phase that was defined by foot-strike (FS) to toe-off (TO) of the right stance limb. FS and TO were automatically determined in accordance with previously published biomechanical methods [39], [40], [41] (calibrated $F_{z}$ rising above $20 \mathrm{~N}$ for $0.025 \mathrm{~s}$, and subsequently falling below $10 \mathrm{~N}$ ), which facilitated stance phase normalization by cubic spline interpolation. The PLS prototype was used to inform specific inclusion time-bases. Consequently, trajectory data was scaled from minus $66 \%$ before FS to TO (125 samples) and force plate data minus $16 \%$ before FS to TO (700 samples). Despite its smaller time-base, the larger number of force plate scaled samples reflected its higher relative data capture frequency compared with marker trajectories. Experimentally derived filters were used to eject duplicate and invalid capture samples (based upon marker trajectories $z, x$ for relevant calcaneus markers; GRF/Ms $F_{z}$ and $M_{z}$ ), regardless of earlier filtering (raw or processed analog outputs), whether the movement was planned or unplanned, FS technique, stepping crossover or regular. Movement trials passing all tests were admitted to the 


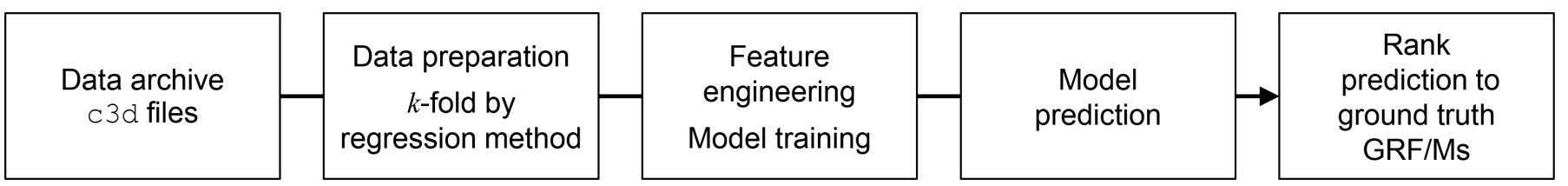

Fig. 2. Study overall design.
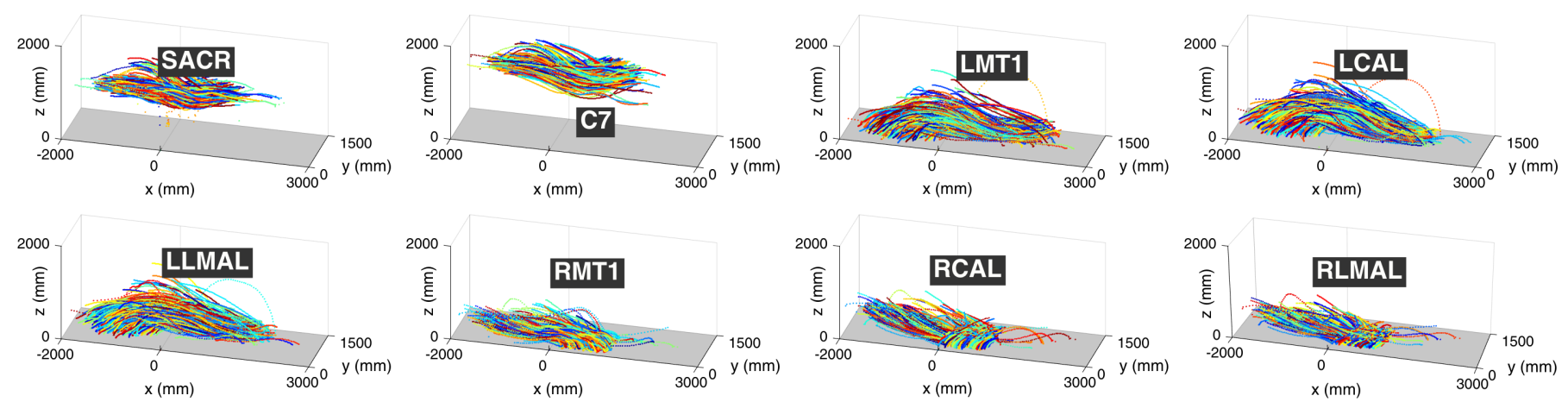

Fig. 3. Training-set eight marker trajectories, sidestep left movement type (off right limb), combined 1,884 predictor samples.

data-set using predictor $X$ and response $y$ array formats typical of multivariate regression [28]. For information only, the final proportions of data-set participants were male $59.1 \%$, female $40.9 \%$, height $1.77 \pm 0.098 \mathrm{~m}$, and mass $73.9 \pm 14.5 \mathrm{~kg}$.

\section{Feature engineering and model training}

A number of $\mathrm{CNN}$ regression models were trained from a random shuffle and split of the data-set using a single fold (training:test). An 80:20 split was used for all methods except GoogLeNet for which a 90:10 ratio was required due to resource limits. Within time constraints, but to check for overfitting [42], one model was tested over 5 -folds whereby each member of the data-set was a participant of each of the five test-sets only once. The single movement type sidestep left was selected, both for its task complexity (i.e. non-planar) but also due to its relevance to knee injury. The total number of sidestep left trials from the archive which were successfully admitted to the data-set was 2,355, which translated to a training-set of 1,884 and a test-set of 471 samples. The large drop-off from the original archive was dominated by incomplete marker labels $(85.5 \%)$.

A prototype had earlier been developed using a number of PLS variants, in which R-spls Sparse SIMPLS was demonstrated as the strongest PLS method for this data and is included here for comparison [28], [27].

CNN network learning speed is increased when the data structures follow a funnel topology, with more input predictor features than those of the output response. Due to its analog technology, and higher sensitivity requirements, force plate data is captured at a higher frequency than motion trajectory data which resulted in more output response features than predictor inputs. Therefore, a variety of dimensionality reduction protocols were assessed including Principal Component Analysis (PCA), Fast Fourier Transform (FFT), and PLS [43], [44]. PCA with tuning threshold $t 0.999$ was selected for its compression accuracy with this data type, which for example with CaffeNet translated to 113 internal components and a ceiling of $r\left(F_{\text {mean }}\right) 0.9997$ and $r\left(M_{\text {mean }}\right) 0.9979$.

To be presented to the $\mathrm{CNN}$ for fine-tuning, the trainingset input marker trajectories were converted from their native spatio-temporal state to a corresponding set of static images [45], [46] (Figure 4). The coordinates of each marker $(x, y, z)$ were mapped to the image additive color model $(R, G, B)$, the eight markers to the image width, and the 125 samples to the image height. CaffeNet for example requires input images to have dimensions $227 \times 227$ pixels, and the resultant $8 \times 125$ image was warped to suit the particular $\mathrm{CNN}$ model using cubic spline interpolation. By freezing space and time, finetuning could be achieved from CNN models pre-trained on image data at scale, which means the new models were able to learn despite the relatively low sample sizes and without the complexity of Long-Term Short Memory (LSTM) layers. Finetuning networks were selected based on their performance and proximity of original training to the current investigation, therefore those trained on the ImageNet classification problem such as AlexNet, CaffeNet and GoogLeNet were apparent candidates (e.g. CaffeNet having been trained on 1.3 million ImageNet images and 1,000 object classifications). Because of its deeper layers relative to available hardware resources, it was necessary to throttle GoogLeNet via smaller batch sizes, and a 90:10 training:test split, processing limitations which prohibited investigation of additional dense networks. Waveform output (rather than the more common object classification) was achieved by replacing the last SoftMax loss with Euclidean loss thereby turning the classifier into a multivariate regression network [47], [48]. All fine-tuning was carried out with the network output reduced via PCA. Additionally, for CaffeNet, the training-set output GRF/M data was deinterlaced into its component waveforms $\left(F_{x}, F_{y}, F_{z}, M_{x}, M_{y}, M_{z}\right)$, and the training process executed six times. 

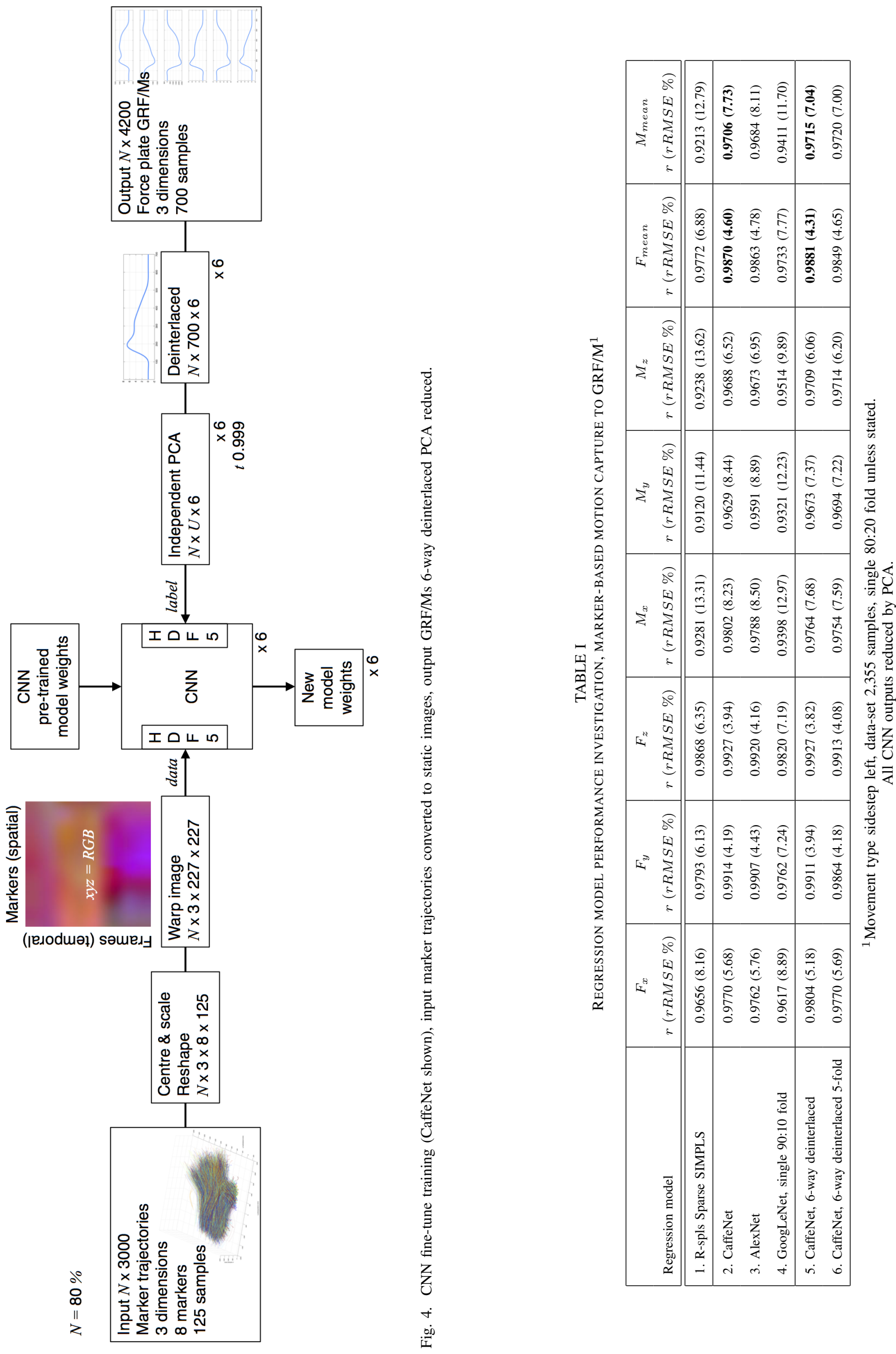


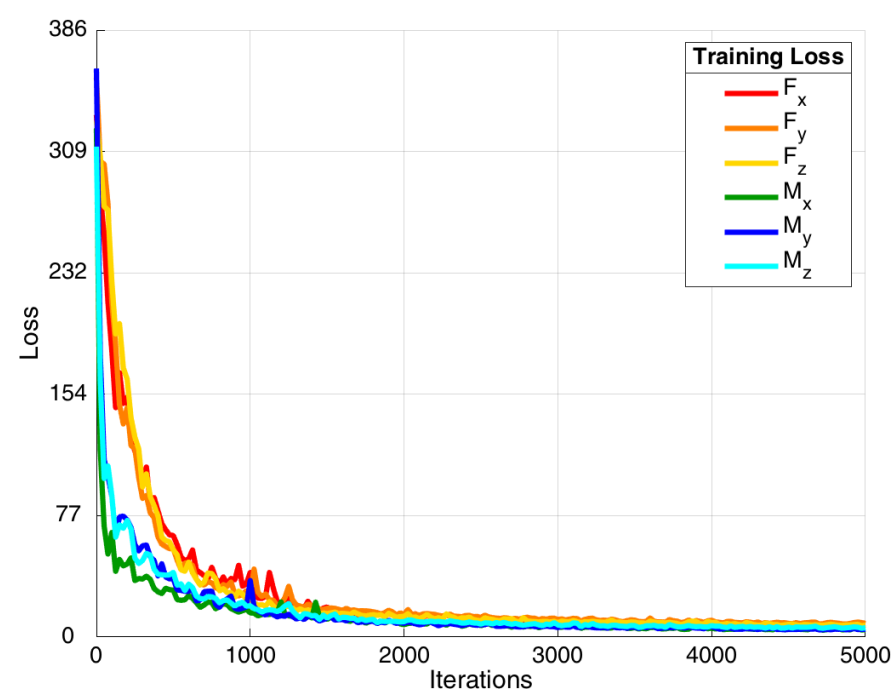

Fig. 5. Learning curve (training loss), CaffeNet, 6-way deinterlaced, six models $F_{x}, F_{y}, F_{z}, M_{x}, M_{y}$, and $M_{z}$.

\section{RESULTS AND DISCUSSION}

The validity of this approach was tested by comparing the difference in accuracy between the ground truth 3D GRF/Ms recorded from the force plate with those predicted by each CNN regression model. To allow comparisons with the literature, two methods of agreement were used, that of correlation coefficient $r$ and relative root mean squared error $r R M S E$ [49]. The mean of the average correlations (or errors) for $F_{x}$, $F_{y}$, and $F_{z}, F_{\text {mean }}$ and similarly for $M_{x}, M_{y}$, and $M_{z}, M_{\text {mean }}$ allowed for ranking of $\mathrm{CNN}$ methods using pairs of numbers (Table II).

The prototype using R-spls Sparse SIMPLS [27] had achieved an average of $r\left(F_{\text {mean }}\right) 0.9772$ and $r\left(M_{\text {mean }}\right)$ 0.9213 ( $r R M S E 6.88$ and $12.79 \%$ ). The closest maximum in the literature using a comparable approach is that of $\mathrm{Oh}$ et al. [24] who reported $r\left(F_{\text {mean }}\right) 0.9647$ and $r\left(M_{\text {mean }}\right) 0.8987$ using a single hidden layer neural network and 48 samples, but for a more simple movement (gait analysis).

Fine-tuning of the selected pre-trained CNN models was investigated, first by training from six output vectors presented as interlaced waveforms and the single resultant array reduced by PCA. Here, CaffeNet proved the most successful with $r\left(F_{\text {mean }}\right) 0.9873$ and $r\left(M_{\text {mean }}\right) 0.9704$ (shown bolded in Table II $r R M S E 4.60$ and $7.73 \%$ ), with AlexNet in close proximity $r\left(F_{\text {mean }}\right) 0.9864$ and $r\left(M_{\text {mean }}\right) 0.9685$ (rRMSE $4.78,8.11 \%)$. This ranking was expected given CaffeNet was designed to improve on AlexNet (by its reversal of the pooling and normalization layers). GoogLeNet came third in this analysis $r\left(F_{\text {mean }}\right) 0.9733, r\left(M_{\text {mean }}\right) 0.9367$ ( $r R M S E$ $7.77,11.70 \%)$, however possibly unthrottled with additional GPU resources, it may return improved results on those presented here. All the fine-tune models achieved significant improvement on the ground reaction moments $r\left(M_{\text {mean }}\right)$ when compared to R-spls Sparse SIMPLS (CaffeNet $+5.4 \%$ ), illustrating the characteristic of $\mathrm{CNNs}$ to achieve stronger relationships in data with greater noise and non-linearity compared with PLS and thus supporting the hypothesis.

The CaffeNet network was further analyzed. Deinterlacing the six output GRF/M waveforms and running PCA (with the same threshold $t$ ) and models for six iterations, resulted in further improved average correlations $r\left(F_{\text {mean }}\right) 0.9880$ and $r\left(M_{\text {mean }}\right) 0.9715$ (shown bolded, $r R M S E 4.31$ and 7.04 $\%)$. Each model was run for 5,000 iterations although the learning curve illustrates the majority of training loss is removed by 2,500 iterations (Figure 5). The training-set, test-set mean, and test-set sample with the corresponding maximum $r\left(F_{\text {mean }}\right)$ are shown (Figure 6). Finally, the CaffeNet PCA deinterlaced method was cross-validated over five k-folds. The similarity of the 5 -fold results $r\left(F_{\text {mean }}\right) 0.9849, r\left(M_{\text {mean }}\right)$ 0.9720 ( $r R M S E 4.65,7.00 \%$ ) with the earlier single-fold experiment indicated overfitting had been avoided.

A limitation of this approach is that despite data preparation best efforts, the method relied on the integrity of the original motion capture and force plate calibration and data capture in order to train an accurate CNN model. In all models investigated, PLS included, the strongest agreement with ground truth was demonstrated in the vertical $\left(F_{z}\right)$ explained by the influence of mass and corresponding greater deviation on which to associate.

\section{CONCLUSIONS}

Using the Caffe deep learning framework, multivariate regression of marker-based motion capture to 3D ground reaction forces and moments was compared via five CNN pretrained models, each tested with sidestep left, this movement type being the most prevalent in the investigation's 17-year data archive. Tactical feature engineering techniques were used to compress spatio-temporal data and thus allow finetuning from three pre-trained CNNs (CaffeNet, AlexNet, and GoogLeNet). By leveraging the big data of the ImageNet database, the authors were able to deliver ground-breaking results from only 2,355 original data capture trials. This should encourage researchers who may think they do not have enough data for deep learning. For this movement-to-GRF/M application, this magnitude of trials could readily be captured with one sports team over a single training season.

The CaffeNet pre-trained CNN model, using dimensionality reduced and deinterlaced outputs, achieved the strongest average correlation to ground truth GRF/Ms with $r\left(F_{\text {mean }}\right)$ 0.9881 and $r\left(M_{\text {mean }}\right) 0.9715$ (rRMSE 4.31 and $7.04 \%$ ). The success of using CNN models to predict GRF/M output for a dynamically complex task advances the project beyond the earlier limitations of PLS and already offers the possibility of use-cases for biomechanical analysis where a force plate is undesirable (or unavailable). Driving the CNN model using marker-based motion capture is simply a necessary first step. Subsequent research using kinematics derived from wearable sensors (or even 2D video) will finally unlock the potential to liberate biomechanists from laboratory constraints and make accurate multidimensional in-game player analyses a reality.

\section{ACKNOWLEDGMENT}

This project was partially supported by the ARC Discovery Grant DP160101458 and an Australian Government Research 

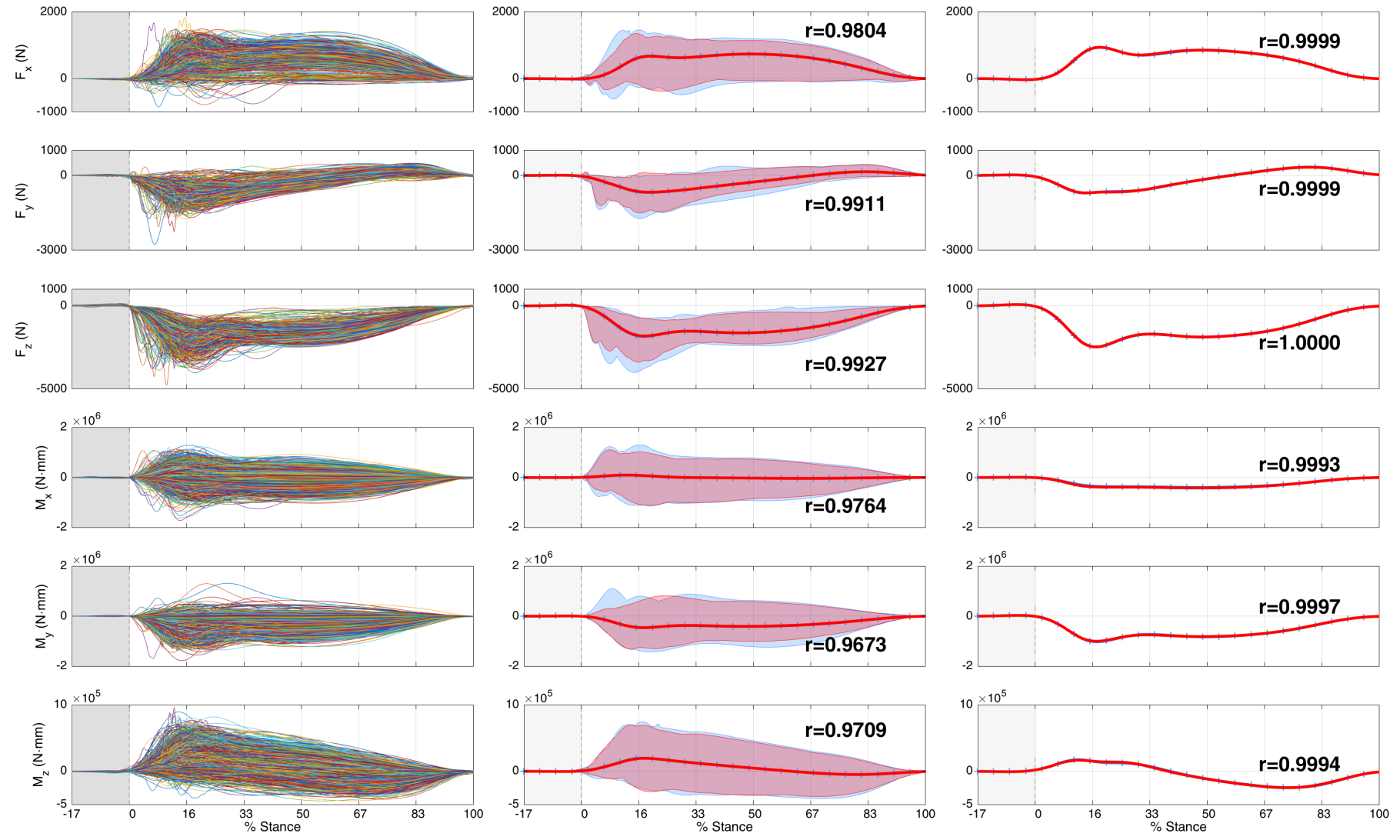

Fig. 6. Left, training-set force plate output $F_{x}, F_{y}, F_{z}, M_{x}, M_{y}$, and $M_{z}, 1,884$ samples versus stance phase. Middle and right, test-set ground-truth GRF/Ms (blue, ticks) and predicted response (red), using CaffeNet, 6-way deinterlaced. Middle, $\mathrm{min} / \mathrm{max}$ range and mean predicted response; right, individual sample with the strongest $r\left(F_{\text {mean }}\right)$.

Training Program Scholarship. We gratefully acknowledge NVIDIA Corporation for the GPU provided through its Hardware Grant Program, and Eigenvector Research for the loan licence of PLS_Toolbox. Portions of data included in this study have been funded by NHMRC grant 400937.

\section{REFERENCES}

[1] L. Chiari, U. Della Croce, A. Leardini, and A. Cappozzo, "Human movement analysis using stereophotogrammetry: Part 2 instrumental errors," Gait \& Posture, vol. 21, no. 2, pp. 197-211, 2005.

[2] B. Elliott and J. Alderson, "Laboratory versus field testing in cricket bowling: A review of current and past practice in modelling techniques," Sports Biomechanics, vol. 6, no. 1, pp. 99-108, 2007.

[3] D. Lloyd, J. Alderson, and B. Elliott, "An upper limb kinematic model for the examination of cricket bowling: A case study of mutiah muralitharan," Journal of Sports Sciences, vol. 18, no. 12, pp. 975-982, 2000.

[4] P. Merriaux, Y. Dupuis, R. Boutteau, P. Vasseur, and X. Savatier, "A study of vicon system positioning performance," Sensors, vol. 17, no. 7, pp. 1591:1-18, 2017.

[5] G. T. Burns, J. D. Zendler, and R. F. Zernicke, "Wireless insoles to measure ground reaction forces: Step-by-step validity in hopping, walking, and running," ISBS Proceedings Archive, vol. 35, no. 1, pp. 295-298, 2017.

[6] T. Liu, Y. Inoue, and K. Shibata, "A wearable ground reaction force sensor system and its application to the measurement of extrinsic gait variability," Sensors, vol. 10, no. 11, pp. 10240-10 255, 2010.

[7] G. S. Faber, I. Kingma, H. M. Schepers, P. H. Veltink, and J. H. Van Dieen, "Determination of joint moments with instrumented force shoes in a variety of tasks," Journal of Biomechanics, vol. 43, no. 14, pp. 2848-2854, 2010.
[8] A. Karatsidis, G. Bellusci, H. M. Schepers, M. de Zee, M. S. Andersen, and P. H. Veltink, "Estimation of ground reaction forces and moments during gait using only inertial motion capture," Sensors, vol. 17, no. 75, pp. 1-22, 2016.

[9] A. Pouliot-Laforte, L. Veilleux, F. Rauch, and M. Lemay, "Validity of an accelerometer as a vertical ground reaction force measuring device in healthy children and adolescents and in children and adolescents with osteogenesis imperfecta type i," Journal of Musculoskeletal and Neuronal Interactions, vol. 14, no. 2, pp. 155-161, 2014.

[10] D. W. Wundersitz, K. J. Netto, B. Aisbett, and P. B. Gastin, "Validity of an upper-body-mounted accelerometer to measure peak vertical and resultant force during running and change-of-direction tasks," Sports Biomechanics, vol. 12, no. 4, pp. 403-412, 2013.

[11] V. Camomilla, E. Bergamini, S. Fantozzi, and G. Vannozzi, "Trends supporting the in-field use of wearable inertial sensors for sport performance evaluation: A systematic review," Sensors, vol. 18, no. 3, pp. 873-922, 2018

[12] R. J. Dallalana, J. H. Brooks, S. P. Kemp, and A. M. Williams, "The epidemiology of knee injuries in english professional rugby union," American Journal of Sports Medicine, vol. 35, no. 5, pp. 818-30, 2007.

[13] C. J. Donnelly, C. Chinnasee, G. Weir, S. Sasimontonkul, and J. Alderson, "Joint dynamics of rear-and fore-foot unplanned sidestepping," Journal of Science and Medicine in Sport, pp. 1-6, 2016.

[14] Y. Shimokochi and S. J. Shultz, "Mechanisms of non-contact anterior cruciate ligament injury," Journal of Athletic Training, vol. 43, no. 4, pp. 396-408, 2008.

[15] T. F. Besier, D. G. Lloyd, J. L. Cochrane, and T. R. Ackland, "External loading of the knee joint during running and cutting maneuvers," Medicine \& Science in Sports \& Exercise, vol. 33, no. 7, pp. 11681175, 2001.

[16] A. R. Dempsey, D. G. Lloyd, B. C. Elliott, J. R. Steele, B. J. Munro, and K. A. Russo, "The effect of technique change on knee loads during sidestep cutting," Medicine \& Science in Sports and Exercise, vol. 39, no. 10, pp. 1765-1773, 2007.

[17] N. Chen, S. Urban, C. Osendorfer, J. Bayer, and P. Van Der Smagt, 
"Estimating finger grip force from an image of the hand using convolutional neural networks and gaussian processes," in 2014 IEEE International Conference on Robotics and Automation (ICRA). IEEE, 2014, Conference Proceedings, pp. 3137-3142.

[18] H. Soo Park and J. Shi, "Force from motion: Decoding physical sensation in a first person video," in Proceedings of the IEEE Conference on Computer Vision and Pattern Recognition, 2016, Conference Proceedings, pp. 3834-3842.

[19] X. Wei and J. Chai, "Videomocap: Modeling physically realistic human motion from monocular video sequences," in ACM Transactions on Graphics (TOG), vol. 29. ACM, 2010, Conference Proceedings, p. 42.

[20] R. Fluit, M. S. Andersen, S. Kolk, N. Verdonschot, and H. F. Koopman, "Prediction of ground reaction forces and moments during various activities of daily living," Journal of Biomechanics, vol. 47, no. 10, pp. 2321-2329, 2014.

[21] J. Yang, B. Howard, A. Cloutier, and Z. J. Domire, "Vertical ground reaction forces for given human standing posture with uneven terrains: Prediction and validation," IEEE Transactions on Human-Machine Systems, vol. 43, no. 2, pp. 225-234, 2013.

[22] T. Sim, H. Kwon, S. E. Oh, S.-B. Joo, A. Choi, H. M. Heo, K. Kim, and J. H. Mun, "Predicting complete ground reaction forces and moments during gait with insole plantar pressure information using a wavelet neural network," Journal of Biomechanical Engineering, vol. 137, no. 9, pp. $091001: 1-9,2015$.

[23] C. Richter, E. King, E. Falvey, and A. Franklyn-Miller, "Supervised learning techniques and their ability to classify a change of direction task strategy using kinematic and kinetic features," Journal of Biomechanics, pp. 1-29, 2017 (in press).

[24] S. E. Oh, A. Choi, and J. H. Mun, "Prediction of ground reaction forces during gait based on kinematics and a neural network model," Journal of Biomechanics, vol. 46, no. 14, pp. 2372-2380, 2013.

[25] A. Choi, J.-M. Lee, and J. H. Mun, "Ground reaction forces predicted by using artificial neural network during asymmetric movements," International Journal of Precision Engineering and Manufacturing, vol. 14, no. 3, pp. 475-483, 2013

[26] T. De Bie, N. Cristianini, and R. Rosipal, Eigenproblems in pattern recognition. Springer-Verlag Berlin Heidelberg, 2005, pp. 129-167.

[27] W. R. Johnson, A. Mian, D. Lloyd, C. J. Donnelly, and J. Alderson, "Predicting athlete ground reaction force and moments from motion capture," Medical \& Biological Engineering \& Computing, vol. (in press), pp. 1-9, 2018

[28] H. Chun and S. Kele, "Sparse partial least squares regression for simultaneous dimension reduction and variable selection," Journal of the Royal Statistical Society: Series B (Statistical Methodology), vol. 72, no. 1, pp. 3-25, 2010.

[29] Y. Jia, E. Shelhamer, J. Donahue, S. Karayev, J. Long, R. Girshick, S. Guadarrama, and T. Darrell, "Caffe: Convolutional architecture for fast feature embedding," arXiv preprint arXiv:1408.5093, 2014

[30] M. Abadi, A. Agarwal, P. Barham, E. Brevdo, Z. Chen, C. Citro, G. S. Corrado, A. Davis, J. Dean, and M. Devin, "Tensorflow: Large-scale machine learning on heterogeneous distributed systems," arXiv preprint arXiv:1603.04467, 2016.

[31] R. Collobert, K. Kavukcuoglu, and C. Farabet, "Torch7: A matlab-like environment for machine learning," in BigLearn, NIPS Workshop, 2011, Conference Proceedings, pp. 1-6.

[32] A. Ichinose, A. Takefusa, H. Nakada, and M. Oguchi, "Pipeline-based processing of the deep learning framework caffe," in Proceedings of the 11th International Conference on Ubiquitous Information Management and Communication. ACM, 2017, Conference Proceedings, pp. 97:1-8.

[33] A. Krizhevsky, I. Sutskever, and G. E. Hinton, "Imagenet classification with deep convolutional neural networks," in Advances in Neural Information Processing Systems, 2012, Conference Proceedings, pp. 10971105.

[34] C. Szegedy, W. Liu, Y. Jia, P. Sermanet, S. Reed, D. Anguelov, D. Erhan, V. Vanhoucke, and A. Rabinovich, "Going deeper with convolutions," in Proceedings of the IEEE Conference on Computer Vision and Pattern Recognition, 2014, Conference Proceedings, pp. 1-9.

[35] S. H. Collins, P. G. Adamczyk, D. P. Ferris, and A. D. Kuo, "A simple method for calibrating force plates and force treadmills using an instrumented pole," Gait \& Posture, vol. 29, no. 1, pp. 59-64, 2009.

[36] T. F. Besier, D. L. Sturnieks, J. A. Alderson, and D. G. Lloyd, "Repeatability of gait data using a functional hip joint centre and a mean helical knee axis," Journal of Biomechanics, vol. 36, no. 8, pp. $1159-1168,2003$

[37] A. Barre and S. Armand, "Biomechanical toolkit: Open-source framework to visualize and process biomechanical data," Computer Methods and Programs in Biomedicine, vol. 114, no. 1, pp. 80-87, 2014.
[38] R. C. Team, R: A language and environment for statistical computing. Vienna, Austria: R Foundation for Statistical Computing, 2016. [Online]. Available: http://www.R-project.org

[39] C. E. Milner and M. R. Paquette, "A kinematic method to detect foot contact during running for all foot strike patterns," Journal of Biomechanics, vol. 48, no. 12, pp. 3502-3505, 2015.

[40] O. Tirosh and W. Sparrow, "Identifying heel contact and toe-off using forceplate thresholds with a range of digital-filter cutoff frequencies," Journal of Applied Biomechanics, vol. 19, no. 2, pp. 178-184, 2003.

[41] C. M. OConnor, S. K. Thorpe, M. J. OMalley, and C. L. Vaughan, "Automatic detection of gait events using kinematic data," Gait \& Posture, vol. 25, no. 3, pp. 469-474, 2007.

[42] P. Domingos, "A few useful things to know about machine learning," Communications of the ACM, vol. 55, no. 10, pp. 78-87, 2012.

[43] K. Pearson, "On lines and planes of closest fit to systems of points in space," The London, Edinburgh, and Dublin Philosophical Magazine and Journal of Science, vol. 2, no. 11, pp. 559-572, 1901.

[44] M. Heideman, D. Johnson, and C. Burrus, "Gauss and the history of the fast fourier transform," IEEE ASSP Magazine, vol. 1, no. 4, pp. 14-21, 1984.

[45] Q. Ke, M. Bennamoun, S. An, F. Sohel, and F. Boussaid, "A new representation of skeleton sequences for $3 \mathrm{~d}$ action recognition," arXiv preprint arXiv:1703.03492, 2017.

[46] Y. Du, Y. Fu, and L. Wang, "Skeleton based action recognition with convolutional neural network," in Pattern Recognition (ACPR), 2015 3rd IAPR Asian Conference on. IEEE, 2015, Conference Proceedings, pp. $579-583$.

[47] Z. Niu, M. Zhou, L. Wang, X. Gao, and G. Hua, "Ordinal regression with multiple output CNN for age estimation," in Proceedings of the IEEE conference on computer vision and pattern recognition, 2016, Conference Proceedings, pp. 4920-4928.

[48] MathWorks. (2018) Train convolutional neural network for regression. [Online]. Available: https://au.mathworks.com/help/nnet/ examples/train-a-convolutional-neural-network-for-regression.html

[49] L. Ren, R. K. Jones, and D. Howard, "Whole body inverse dynamics over a complete gait cycle based only on measured kinematics," Journal of Biomechanics, vol. 41, no. 12, pp. 2750-2759, 2008. 